\title{
Fabrication of Twisted Nematic Structure in Phase Separated Composite Films
}

\author{
Hak-Rin Kim \\ Jong-Wook Jung \\ You-Jin Lee \\ Jae-Hoon Kim \\ Department of Electronics and Computer Engineering, \\ Hanyang University, Seoul, Korea
}

We investigated the liquid crystal (LC) alignment effect of the polymerized interface in the phase-separated composite film (PSCOF). Since the polymer morphology after phase separation depends highly on the LC ordering during phase separation, the azimuthal anchoring strength of the LC/polymer interface increased with highly ordered LCs. Using anchoring capability induced by imprinting effect of molecular ordering, we demonstrated a twisted nematic structure in PSCOF by application of in-plane electric fields during phase separation.

Keywords: azimuthal anchoring; liquid crystal alignment; molecular ordering; phaseseparated composite film; phase separation; twisted nematic structure

\section{INTRODUCTION}

Electro-optic (EO) properties of liquid crystal (LC) devices depend highly on the LC configuration in bulk. To get desired performances by manipulating birefringent LC layer, several types of alignment techniques have been proposed. With a conventional alignment method, a LC cell should be prepared by a pair of alignment layer in general. Recently, a new type of fabrication method requiring only single alignment treatment was reported, which is referred to as a phase-separated composite film (PSCOF) method [1-3]. The PSCOF

This work was supported in part by Korea Research Foundation Grant No. KRF2004-005-D00165.

Address correspondence to Prof. Jae-Hoon Kim, Department of Electronics and Computer Engineering, Hanyang University, 17 Haengdang-dong, Seongdong-gu, Seoul 133-791, Korea (ROK). E-mail: jhoon@hanyang.ac.kr 
structure is composed of a stack of a thin LC and polymer layer, prepared by an anisotropic phase separation of LCs from its solution in prepolymers [4]. It is known that such properties of PSCOF cell as the good mechanical stability and the flexibility of polymer layer itself have advantages in fabricating flexible LCDs [1,5-7]. Using these features, we have demonstrated a LCD with single substrate before [5]. However, the LC geometry produced by the conventional PSCOF method is limited to a uniform homogeneous structure since the LC orientation is determined only by the rubbing direction of the single alignment layer and the phase separation process is executed in isotropic phase of LC, which is existing problem of the PSCOF to be solved [1,2].

In this work, we proposed a fabrication method which makes several types of LC geometries available in PSCOFs. This was accomplished by inducing anisotropy in formation of polymer chains during phase separation. To get a uniform and a highly anisotropic polymer interface in the PSCOF system, the conventional phase separation process was modified into two steps of phase separation where temperature and resultant LC ordering were controlled to have a different condition in each step. It was observed that the LC ordering during phase separation had a great influence on the polymer chain formation. The LC anchoring effect by imprinted ordering on the polymer layer was sufficient for competing with the elastic energy in the bulk and we demonstrated a twisted nematic (TN) structure in PSCOF by application of in-plane electric fields during phase separation in nematic phase.

\section{EXPERIMENTAL}

The scheme of our PSCOF cell structure is demonstrated in Figure 1. The cell was made using two glass substrates. One of the substrate has in-plane electrodes prepared by etching ITO in chevron pattern with $100 \mu \mathrm{m}$ wide and $100 \mu \mathrm{m}$ separation. Then, the patterned substrate was spin-coated with $1 \mathrm{wt} \%$ of a Nylon 6 in trichloroethanol. After drying, the substrate was rubbed in a direction as shown in Figure 1. The other substrate had no electrode and no alignment layer. The cell gap was maintained by $5 \mu \mathrm{m}$ glass spacers. The material used for phase separation were E48 (E. Merck) for a nematic LC (NLC) and UV curable epoxy NOA 65 (Norland) for a prepolymer. The phase transition sequence of $\mathrm{E} 48$ is crystal $\rightarrow\left(-19^{\circ} \mathrm{C}\right) \rightarrow$ nematic $\rightarrow\left(87^{\circ} \mathrm{C}\right) \rightarrow$ isotropic. The NLC/prepolymer mixture in a ratio of 50:50 was introduced into our cells at $100^{\circ} \mathrm{C}$.

The anisotropic phase separation shown in Figure 1 was initiated by irradiating UV light normal to the untreated substrate. The UV 


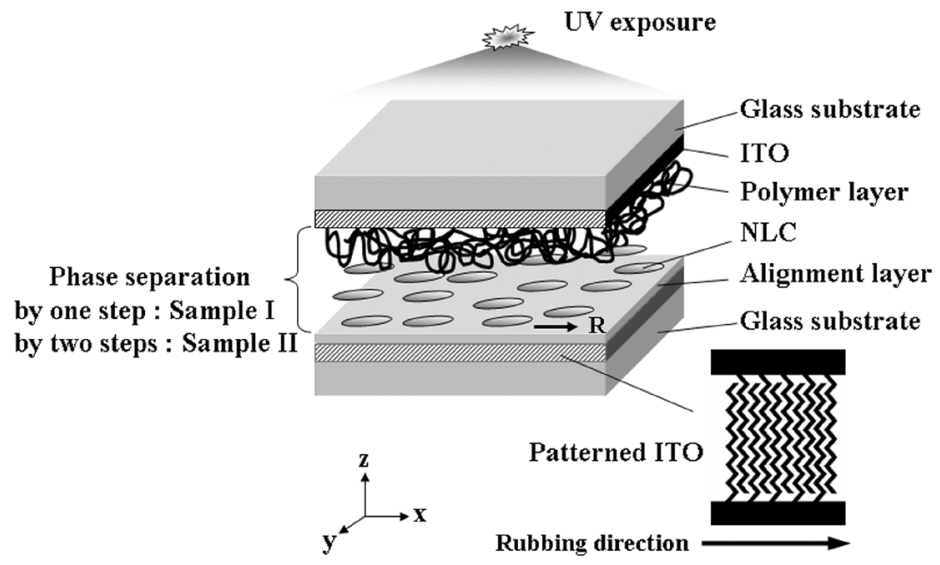

FIGURE 1 Schematic of PSCOF structure. Two types of PSCOF cells are prepared; Sample I is fabricated by phase separation in isotropic phase of the LCs without an applied voltage. Sample II is fabricated by phase separation in nematic phase of the LCs with in-plane electric field.

lamp was operated in an electrical power of $400 \mathrm{~W}$. In our UV intensity condition, low rate of phase separation is created, which is one of essential fabrication conditions for uniform PSCOF structure [2]. In the conventional fabrication condition for PSCOF, the phase separation was executed by single step of UV exposure in isotropic state of a NLC. Such temperature condition is another key parameter in getting uniform bilayer system in PSCOF [2,4]. At higher temperature, the NLC molecules and the prepolymers can more freely diffuse in the opposite direction with each other, that is important in our anisotropic phase separation.

To compare our fabrication method with the conventional one, we prepared two types of samples. Except phase separation condition, the conditions including the rubbing direction with respect to the patterned electrode were identical between two cells. The first one (Sample I) was fabricated by UV exposure at $100^{\circ} \mathrm{C}$ without bias field for 30 min following the conventional PSCOF method. The second one (Sample II) was fabricated by two steps of UV exposures with varying temperature condition at each step. At first UV exposure of Sample II, the phase separation was executed at $100^{\circ} \mathrm{C}$ without bias field for about $10 \mathrm{~min}$. The first UV exposure was positively necessary also in our fabrication procedures to promote uniform anisotropic phase separation. Then, the cell was cooled down to $40^{\circ} \mathrm{C}$ and then further exposed to UV with bias field of $2 \mathrm{~V} / \mu \mathrm{m}(1 \mathrm{kHz}$, square wave) for 


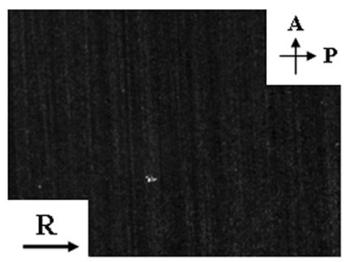

(a)

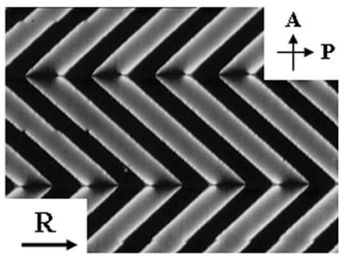

(c)

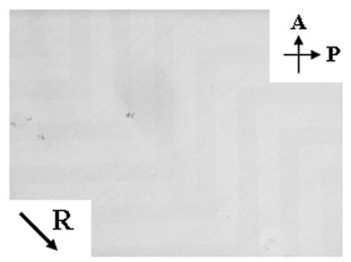

(b)

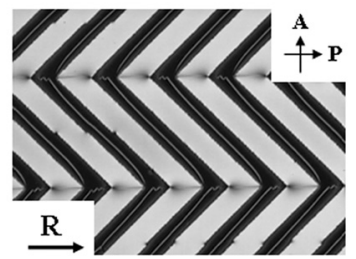

(d)

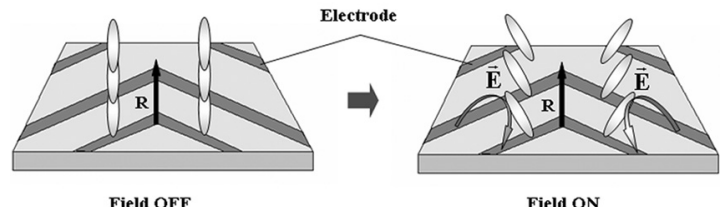

(e)

FIGURE 2 Microscopic images of Sample I between the crossed polarizers; (a) and (b) are the alignment textures obtained when the rubbing direction is parallel and rotated by $45^{\circ}$ with respect to the polarizer axis, respectively. (c) and (d) are the textures with electric field of $0.5 \mathrm{~V} / \mu \mathrm{m}$ and $1 \mathrm{~V} / \mu \mathrm{m}$, respectively when the rubbing direction is parallel to the polarizer axis. (e) shows the NLC ordering after phase separation without or with an applied voltage.

$30 \mathrm{~min}$. Notice that the phase of our LC is nematic at the second phase separation procedure. After the second phase separation, an anisotropic anchoring of a NLC was created at the NLC/polymer interface.

\section{RESULTS AND DISCUSSION}

Figure 2 shows polarizing microscopic images of Sample I between the crossed plarizers, where (a) and (b) were obtained in the absence of an applied voltage and (c) and (d) were obtained in the presence of an applied voltage. When the cell that was initially parallel to one of polarizers was rotated by $45^{\circ}$, the image of Sample I turned from dark state to bright state, which meant that the LCs were homogeneously aligned along the rubbing direction in a uniformly phase-separated 
(a)

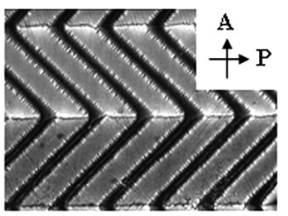

(c)

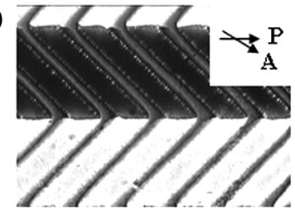

uv

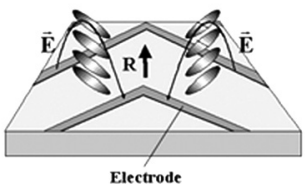

(e) (b) $<-<, c, A$

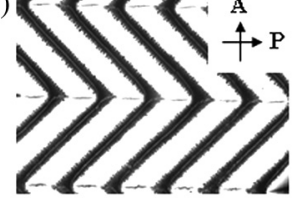

(d)

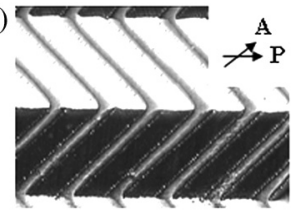

field off

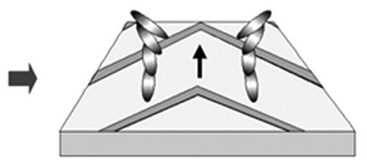

(f)

FIGURE 3 Polarizing microscopic images of the Sample II. (a), (c), and (d) are obtained in the absence of an applied voltage and (b) is obtained in the presence of an applied voltage, $2 \mathrm{~V} / \mu \mathrm{m}$, in different geometries of polarizers. (e) and (f) show the NLC ordering during phase separation in the presence of an applied voltage and after UV exposure in the absence of an applied voltage, respectively.

state as shown in Figure 2(e). As an applied voltage was increased, transmittance between the in-plane electrodes increased due to LC reorientation along the field direction. In the conventional PSCOF case, the NLC/polymer interface is isotropic in the azimuthal anchoring since there is no imprinting effect by the LC molecules and the polymer chains at the interface has random distribution.

The polarizing microscopic images of Sample II obtained between the crossed polarizers with and without a bias voltage are given in Figures 3(a) and (b), respectively. Notice that the LC texture in Figure 3(a) did not show dark state even in the off-state different from that of Sample I. This means that a significant change took place in the phase-separated polymer during the second UV exposure. To identify the NLC geometry between the alignment layer and the phase-separated polymer layer, the polarization direction of the exit light through Sample II was monitored by rotating the transmission axis of the analyzer in the absence of an applied voltage with keeping the incident polarization parallel to the rubbing direction. In Figures 
(c) and (d), the dark and bright states in each chevron domain with respect to the analyzer axis clearly showed that the NLCs were aligned with $45^{\circ}$ and $-45^{\circ}$ twisted structures with opposite handness at each domain.

Figure 3(e) and (f) show the schematic of the NLC orientation in the presence of an applied voltage during the second phase separation and in the absence of an applied voltage after the phase separation was completed, respectively. The LC orientation at the NLC/polymer interface coincided with the direction of the electric field at the second UV exposure, which means that field-induced LC ordering changed the NLC/polymer interface into an anisotropic alignment layer during the second phase separation. The anchoring capability of the polymer layer along the LC orientation might originate in the polymer chain ordering during phase separation. The result of Sample II showed that this imprinted anisotropy was strong enough to compete with the elastic energy of LC even in our thin cell gap of $2.5 \mu \mathrm{m}$.

\section{CONCLUSIONS}

We proposed the phase separation method for varying the LC geometry in the PSCOF cell. By controlling temperature condition and the LC ordering at two steps of phase separation, we could fabricate the uniform PSCOF cell of which polymer layer could align the LCs in a predetermined direction since the bulk LC ordering was imprinted to the morphology of the NLC/polymer interface during phase separation. The twisting structure of the LC could be easily realized by application in-plane electric fields during our phase separation procedures. It is expected that our method would be a useful alignment method for enhancing electro-optic performance of a $\mathrm{LC} /$ polymer composite system.

\section{REFERENCES}

[1] Vorflusev, V. \& Kumar, S. (1999). Science, 283, 1903.

[2] Qian, T., Kim, J.-H., Kumar, S., \& Taylor, P. L. (2000). Phys. Rev. E., 61, 4007.

[3] Wang, Q. \& Kumar, S. (2005). Appl. Phys. Lett., 86, 071119.

[4] Krongauz, V., Schmelzer, E., \& Yohahannan, R. (1991). Polymer, 32, 1654.

[5] Kim, I., Kim, J.-H., Kang, D., Agra-Kooijman, D. M., \& Kumar, S. (2002). J. Appl. Phys., 92, 7699.

[6] Jung, J.-W., Park, S.-K., Kwon, S.-B., \& Kim, J.-H. (2004). Jpn. J. Appl. Phys., 43, 4269.

[7] Jang, S.-J., Jung, J.-W., Kim, H.-R., Jin, M., \& Kim, J.-H. (2005). Jpn. J. Appl. Phys., 44,6670 . 